\title{
CRAB-LIKE SUPERNOVA REMNANTS
}

\author{
R.H. Becker \\ Physics Department \\ University of California \\ Davis, CA 95616 \\ and \\ Institute of Geophysics and Planetary Physics \\ Lawrence Livermore National Laboratory
}

ABSTRACT. Crab-like SNR are markers for recently formed pulsars. The current catalog of 15 objects allows a direct measure of the space distribution of pulsars, the beaming factor of pulsars, and the energetics at young pulsars. The $15 \mathrm{Crab}-\mathrm{like}$ SNR are equally divided between those with surroundings shells and those without. No other properties appear to correlate with the presence or lack of a shell. The ratio of 10 to 1 between all remnants and Crab-like remnants has important implications for the formation of pulsars.

\section{INTRODUCTION}

Although we know of hundreds of pulsars, only four of them are demonstrably younger than $\sim 10^{4}$ years: the Crab pulsar, the Vela pulsar, MSH15-52, and 0540-693. This is a small number in comparison with the $\sim 150$ known galactic supernova remnants (SNR). It even lags behind the number of galactic historical supernovae. The uncertainty in the selection effects which hinder detection of pulsars makes it difficult to extrapolate from the four observed young pulsars to the population at large.

We can extend our knowledge of young pulsars by studying the associated population of Crab-like SNR of which $\sim 15$ are currently known in the galaxy. In this paper, I will present the current status of the observational data for Crab-like SNR and indicate what the implications are for young pulsars.

\section{A CATALOG}

Studies of Crab-like SNR have developed rather slowly over the past 15 years with a fair amount of confusion and backtracking. The IAU Symp. 46 on The Crab Nebula held in 1970 only contains reference to 3 Crab-like objects. At that time, Minkowski (1971) pointed out 
that "The frequency of pulsars .... is indeed in complete agreement with the observed frequency of supernovae. One might wonder why there is not a pulsar in every supernova remnant." He could well have asked the same question in regard to Crab-like SNR. The absence of pulsars has generally been explained in terms of beaming, high dispersion measures, and low sensitivity. The absence of Crab-like SNR remains unsolved.

By 1982 , at the IAU Symposium 非101 entitled SNR and Their $x$-ray Emission, the number of proposed Crab-like SNR had grown to $\sim 20$ and two subclasses had emerged (Weiler 1971, Becker 1971). In addition to sources similar to the Crab Nebula, some Crab-like objects had been discovered within more traditional SNR shells. For the rest of this paper I will refer to the two subclasses as "Crabs" and "Crab Shells". Of the $\sim 20$ aforementioned objects, 8 were Crabs and 12 were $\mathrm{Crab}$ Shells.

In retrospect, many of the identifications assert in 1982 were premature and today I would put the number of secure identifications at $15,8 \mathrm{Crabs}$ and $7 \mathrm{Crab}$ Shells. (This count excludes extragalactic members of the class). In Table I, I list the 15 sources and also

TABLE I. Catalog of Crab-like SNR

\section{Crabs}

G20.0+0.2 (Becker \& Helfand 1985)

G21.5-0.9 (Davelaar et al. 1986)

G54.1+0.3 (Reich et al. 1985)

G74.9+1.2 (Wilson 1980)

G130.7+3.1 (Green 1986)

G184.6-5.8 (Velusamy 1985)

G291.0-0.1 (Wilson 198 )

G328.4+0.2 (Caswell et al. 1980)

Crab Shells

G0.9+0.1 (Helfand \& Becker 1986)

G24.7+0.6 (Reich et al. 1984)

G29.7-0.3 (Becker \& Helfand 1984)

G263.9+2.8 (Harnden et al. 1985)

G320.4-1.2 (Seward et al. 1984)

G326.3-1.8 (Milne et al. 1985)

G351.2+0.1 (Becker \& Helfand 1986)

Other SNR with compact sources

G27.4+0.0 (Kriss, G. et al. 1985)

G39.7-2.0 (Downes et al. 1986)

G68.9+2.8 (Strom \& Blair 1985)

G109.1-1.0 (Hughes et al. 1984)

G332.4-0.4 (Tuohy et al. 1983) 
include 5 addition SNR which appear to contain neutron stars which are not pulsars.

Of the 15 Crab-like objects 3 contain pulsars and a fourth contains an unresolved $x$-ray source at its center. Does this imply a beaming factor of 4? More likely 4 should be taken as a lower limit in so far as most of these sources have not undergone exhaustive searches for pulsars. Such searches should be of the upmost importance for they would permit a direct measure of the beaming factor of young pulsars.

\section{CRABS AND CRAB SHELLS}

We do not know if Crabs and Crab Shells are truly two distinct phenomena with different types of progenitors or if they instead a continuous gradation of core vs. shell luminosity. In figure 1, I show a histogram of the number of Crab Shells as a function of the ratio of core to shell luminosities. In addition I have plotted the lower limit of the ratio for two Crabs, the Crab Nebula (Velusamy 1983) and 3C58 (Reynolds and Allen 1985). Among the Crab Shells, the ratio spans 3 orders of magnitude while the inclusion of Crabs brings the range to over 5 orders of magnitude. The lower end of the range may be an observational limit. It would be difficult to observe a Crablike component which only contributes $<.001$ of the total luminosity. I see no compelling rationale for distinguishing between the two kinds of Crab-like SNR based on the presence or absence of a shell.

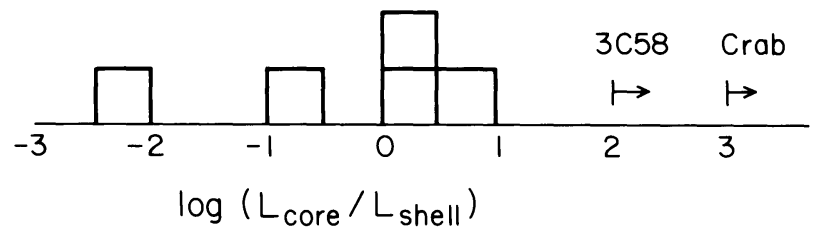

Figure 1. Ratio of core luminosity to shell luminosity.

Figure 2 displays the distribution in galactic latitude of Crabs and Crab Shells. In fact, they are surprising similar in so far as the vastly different selection effects involved in their discovery. The Crab Shells are surrounded by steep spectrum shells which are readily discernible at low frequencies while the Crabs have flat spectra and are easily confused with HII regions. In Figure 3 , the $\mathrm{z}$-distribution of Crabs and Crab Shells are plotted. Again we see little significant difference. The distribution of $B$ stars has been plotted as well. There may be some evidence that Crab-like SNR are formed further from the plane than massive stars, the commonly accepted progenitors.

Recently, it has become popular to consider the ratio of $x$-ray to radio luminosity as a way to rank Crab-like objects. In Table II, the 

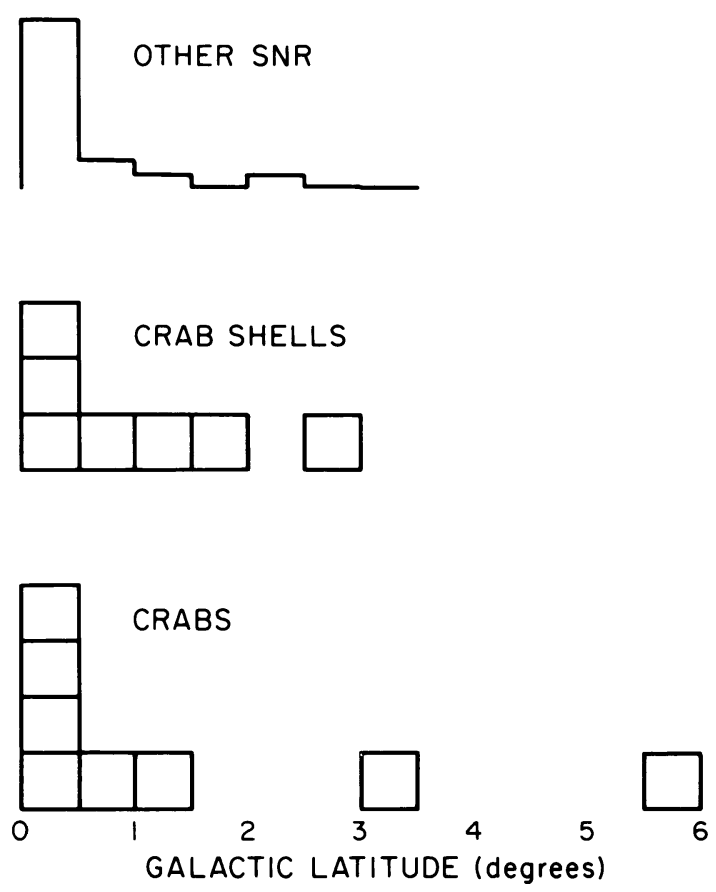

Figure 2. Latitude distribution of Crabs, Crab Shells, and other SNR.
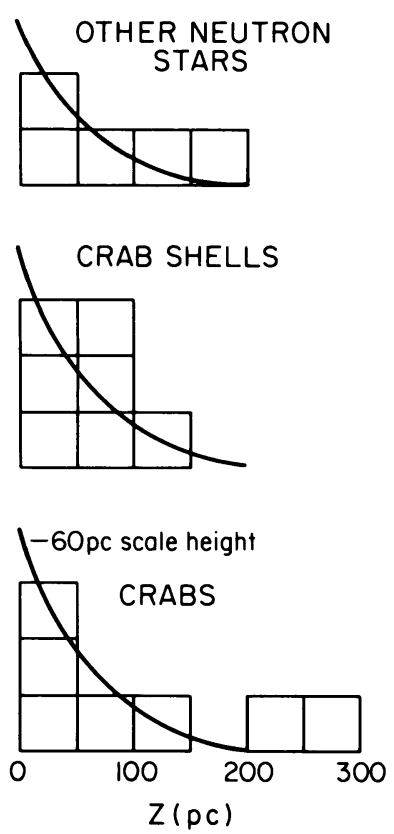

Figure 3. Z-distribution of Crabs, Crab shells, and other SNR containing neutron stars. 
values of $\mathrm{L}_{x} / \mathrm{L}_{\mathrm{R}}$ for Crabs and Crab Shells alike are listed in descending order. Again no clear difference between Crabs and Crab Shells emerge.

TABLE II. Ratio of X-ray to Radio Luminosity for Crab-like SNR

$\begin{array}{lc}\text { Source } & \frac{\mathrm{L}_{x} / \mathrm{L}_{r}}{500} \\ \text { G29.7-0.3 } & 100 \\ \text { Crab } & 15 \\ \text { G21.5-0.9 } & \geq 10 \\ \text { MSH 15-52 } & 1.3 \\ \text { G74.9+1.2 } & 1 \\ \text { G291.0-0.1 } & \leq .5 \\ \text { G0.9+0.1 } & .5 \\ \text { 3c58 } & .1 \\ \text { Vela X } & \end{array}$

We would like to use Crab-like SNR as a means of studying pulsar energetics. This is most reliably done by measuring the $x$-ray flux from Crab-like remnants. Since the $x$-ray emitting electrons rapidly radiate away their energy, the current $x$-ray luminosity should be closely correlated to the energy being put into the acceleration of particles by the pulsar. Ideally, we would then relate this number to the total energy less rate of the pulsar. Helfand (1984) has tabulated the $x$-ray luminosity of Crab-like SNR and several older pulsars and the energy loss rate of the associated pulsar if there is one. It seems that in most cases $1-5 \%$ of the pulsar energy losses are converted into $x$-ray emission over a wide range of pulsar ages. Therefore, we can reasonably estimate pulsar energetics even when the pulsar is not observable.

\section{PULSAR BIRTH RATES}

Previously, pulsar birthrates have been calculated based on pulsar surveys. These estimates depend on the value of the beaming factor used. Since the emission from Crab-like SNR are not beamed, they might provide a less model-dependent birthrate estimate. Presently we know of 15 SNR which show evidence for a young pulsar. This represents $\sim 10 \%$ of the total number of known galactic SNR implying a birthrate only $\sim 20 \%$ of that inferred from pulsar statistics (assuming a beaming factor of 5). How can we reconcile this inconsistency? I will suggest four possible solutions. 


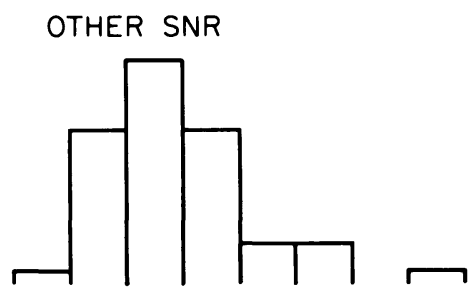

CRAB SHELLS(Core only)

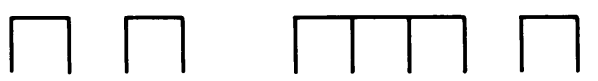

CRABS

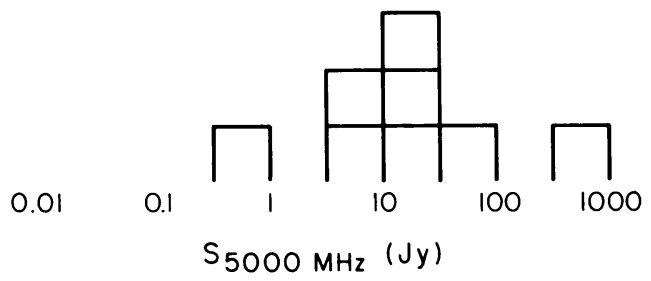

Figure 4. Radio flux at $5000 \mathrm{MHz}$ of Crabs, Crab shells, and other SNR.

The most trivial solution is that there are many more Crab-like SNR yet to be discovered but remain hidden due to observational selection effects. This could be the case if most Crab-like remnants have $6 \mathrm{~cm}$ fluxes below several Janskies. The histograms in Fig. 4 show the distribution of $6 \mathrm{~cm}$ flux for the known Crabs and Crab Shells. Note particularly that the cores of two Crab Shells have fluxes below $0.5 \mathrm{Jy}$. It would be easier to accommodate a large increase in Crab Shells from the known population of shell remnants because this would not change the overall birthrate estimates. The discovery of $\sim 100$ weak Crabs would upset the current equality of SN with SNR birthrates. Alternatively, the small percentage of Crab-like SNR could result if Crab-like SNR have lifetimes short compared to shell remnants. This possibility is in some sense a variant of suggesting that most Crablike remnants are too weak to observe.

Others have suggested that the majority of pulsars are born as slow rotators (period $>0.3 \mathrm{sec}$ ) and hence are never energetic enough to create synchrotron nebula. These conclusions are supported by studying the observed population of old pulsars and evolving them backwards to infer the properties of the initial population. This argument would gain greater support if a slow pulsar were found associated with a SNR. To date, no such occurrence has been found.

Lastly, we could accept the observational result that only $\sim 10 \%$ of SNR contain pulsars. That is to say only $10 \%$ of galactic SN result in the formation of a pulsar. Reconciliation of this result with the 
observed space density of pulsars would require a beaming factor of order unity rather than the nominal value of 5 .

\section{REFERENCES}

Becker, R.H. 1983 in Supernova Remnants and Their X-ray Emission (ed. by Danziger and Gorenstein) D. Reidel, Dordrecht, p. 329.

Becker, R.H. and Helfand, J. 1984 Ap. J. 281, 650.

Becker, R.H. and Helfand, J. 1985 Ap. J. (Letters) 297, L25.

Becker, R.H. and Helfand, J. 1986 in preparation.

Caswell et al. 1980, MNRAS 190, 882.

Davelaar et al. 1986 Ap. J. (Letters) 300, L59.

Downes et al. 1986 MNRAS 218, 393.

Green 1986 MNRAS 218, 533.

Harnden et al. $19 \overline{85}$ Ap. J. $299,828$.

Helfand, D.J. 1984, Adv. Space Res. 3, 29.

Helfand, D.J. and Becker, R.H. 1986 Ap. J., in press .

Hughes et al. 1984, Ap.J. 283, 147.

Kriss, G. et al. 1985 Ap. J. 288, 703.

Milne, D. et al. 1985 Proc. Astron. Soc. Aust. 6, 78.

Minkowski, R. 1971 in The Crab Nebula (ed. by Davies and Smith) D. Reidel, Dordrecht, p. 241.

Reich et al. 1985 Astron. Astrophys. 151, L10.

Reich et al. 1984 Astron. Astrophys. $\overline{133}$, L4.

Reynolds, S.P. and Aller, H. 1985 A.J. 90, 2312.

Seward, F. et al. 1984 Ap. J. 281650.

Strom, R. and Blair, W. 1985 Astron. Astrophys. 149, 254.

Tuohy, et al. 1983 Ap. J. 268, 778 .

Velusamy, T. 1985 in The C $\overline{\mathrm{rab}}$ Nebula and Related Supernova Remnants

(ed. by Kafatos and Henry) Cambridge Univ. Press, p. 115.

Weiler, K.W. 1983 in Supernova Remnants and Their X-Ray Emission (ed.

by Danziger and Gorenstein) D. Reidel, Dordrecht, p. 299.

Wilson, A. 1980 Ap. J. (Letters) 241, L19.

Wilson, A. 1986 Ap.J.

\section{DISCUSSION}

S. Woosley: Is there any case of a pulsar existing in an oxygenrich SNR. Could pulsar-containing remnants all be compositionally like the Crab, i.e., helium-rich and little else? Is the rotation rate of the pulsar especially slow in such cases (if they exist)?

R. Becker: The LMC pulsar 0540-693 is very similar to the Crab in al1 its nonthermal properties but is surrounded by an oxygenrich she11. Many of the other Crab-like nebulae are in remnants that have not been detected optically. 